\title{
ON THE NUMBER OF EIGENVALUES IN THE SPECTRAL GAP OF A DIRAC SYSTEM
}

\author{
by D. B. HINTON, A. B. MINGARELLI, T. T. READ and J. K. SHAW
}

(Received 16th May 1985)

\section{Introduction}

We consider the one-dimensional operator,

$$
L y:=\left(\begin{array}{rr}
0 & -1 \\
1 & 0
\end{array}\right)\left\{y^{\prime}-\left(\begin{array}{cc}
p(x) & c_{1}+V_{1}(x) \\
c_{2}-V_{2}(x) & -p(x)
\end{array}\right) y\right\},
$$

on $0<x<\infty$ with $y=\left(\begin{array}{l}y_{1} \\ y_{2}\end{array}\right)$. The coefficients $p, V_{1}$, and $V_{2}$ are assumed to be real, locally Lebesgue integrable functions; $c_{1}$ and $c_{2}$ are positive numbers. The operator $L$ acts in the Hilbert space $H$ of all equivalence classes of complex vector-value functions $f=\left(\begin{array}{l}f_{1} \\ f_{2}\end{array}\right)$ such that $\int_{0}^{\infty}\left(\left|f_{1}\right|^{2}+\left|f_{2}\right|^{2}\right) d x<\infty$. L has domain $D(L)$ consisting of all $y \in H$ such that $y$ is locally absolutely continuous and $L y \in H$; thus in the language of differential operators $L$ is a maximal operator. Associated with $L$ is the minimal operator $L_{0}$ defined as the closure of $L_{0}^{\prime}$ where $L_{0}^{\prime}$ is the restriction of $L$ to the functions with compact support in $(0, \infty)$.

The singular structure of the coefficients at 0 determine whether $L=L_{0}$ (and hence $L$ is selfadjoint - the limit-point case) or $L$ contains $L_{0}$ properly (the limit-circle case). In the latter case the selfadjoint operators $L_{1}$ generated by $L$ satisfy $L_{0} \subset L_{1} \subset L$ and are determined by imposing a boundary condition at $x=0$ on the elements of $D(L)$. The limit-point and limit-circle terminology arises from the geometric method of Weyl and is discussed in [18]. We use $L_{1}$ to denote a selfadjoint extension of $L_{0}$ in either case. In any event the essential spectra of $L_{0}, L_{1}$, and $L$ coincide. Essential selfadjointness criteria and construction of selfadjoint extensions of $L_{0}$ have been discussed in many papers, e.g. $[1,8,9,10,11,12,20,22,23,24]$.

Under rather general conditions with $p, V_{1}, V_{2}$ "small" at infinity and sufficiently "regular" at 0 , the essential spectrum of $L_{1}$ is $\left(-\infty,-c_{1}\right] \cup\left[c_{2}, \infty\right)$. Results on location of essential spectra are given in $[3,8,9,14,23]$. When the gap $\left(-c_{1}, c_{2}\right)$ contains no essential spectrum of $L_{1}$, it can contain only eigenvalues of $L_{1}$. The purpose of this paper is to obtain conditions on the coefficients which determine if the gap contains finitely or infintely many eigenvalues of $L_{1}$. Since $L_{1}$ is a finite dimensional extension of $L_{0}$, the finiteness of the gap spectra is independent of $L_{1}$. Another problem, also independent of $L_{1}$, is to determine when the gap spectrum is infinite which of the endpoints $-c_{1}$ and $c_{2}$ are cluster points of eigenvalues. While our theorems do not assume the existence of a gap, it is the case of primary interest. 
For $V_{1}=V_{2}$, equation (1.1) arises from the three-dimensional Dirac equation with a spherically symmetric potential after a separation of variables. For the choices $p(x)=k / x$, $V_{1}(x)=V_{2}(x)=z / x$, and $c_{1}=c_{2}=c,(1.1)$ is the radial wave equation in relativistic quantum mechanics for a particle in a field of potential $V(x)=z / x$. When the anomalous magnetic moment of the particle is considered, $p(x)=k / x$ is replaced by $p(x)=k_{1} / x+k_{2} / x^{2}$.

The discreteness of the spectrum of $L_{1}$ in $\left(-c_{1}, c_{2}\right)$ was studied by Birman [3, Section 5]. Using the methods of Birman, Kurbenin [15] gave criteria for the spectrum in the gap to be finite or to be infinite. The work of Kurbenin requires $p(x)=k / x$ and $V_{1}(x)=$ $V_{2}(x)$ to be uniformly bounded on $(0, \infty)$. The one-dimensional results of Kurbenin will follow from the results given below. Further discussion is given in [7, Section 62].

In the three-dimensional Dirac equation with gap $[-1,1]$ and with a regularly growing potential that decays like $|x|^{-m}, 0<m<2$, as $|x| \rightarrow \infty$, Tamura [21] has given asymptotic behavior for the number of eigenvalues in $(0,1-r)$ as $r \rightarrow 0$. Also in the threedimensional case with a parameter $\Lambda$ multiplying the potential, Klaus [13] has given an asymptotic formula for the number of eigenvalues in the gap as $\Lambda \rightarrow \infty$.

In Section 2 below we give conditions for gap spectra to be infinite. By means of oscillation theory we generate a family of second order scalar differential equations for which the oscillation of one of them at 0 or $\infty$ is sufficient to give infinite gap spectra. Section 3 treats the converse problem and gives a comparison second order vector equation whose non-oscillation implies a finite gap spectra. Additional results are given by treating the operator $L$ directly. An interesting corollary of the latter method is a sufficient condition for the length of the gap to be "infinite", i.e. $L_{1}$ has empty essential spectrum. Finally in Section 4, we use a shifting technique which for certain equations permits one to determine which endpoint of a gap is a cluster point of eigenvalues.

We use the following notation: $($,$) represents the inner product in both H$ and $R^{n}$ with corresponding norm \|\|$, I$ is an identity operator or matrix, $\sigma(A)$ is the spectrum of $A$, and if $J$ is an interval, $C_{0}(J)$ is the set of all continuously differentiable 2-vectorvalued functions with compact support contained in $J$.

\section{Infinite gap spectra}

First we consider the square of $L$ given by

$$
L^{2} y=-\left(y^{\prime}+J P y\right)^{\prime}-P\left(J y^{\prime}-P y\right)
$$

where

$$
J=\left(\begin{array}{rr}
0 & -1 \\
1 & 0
\end{array}\right), \quad P=\left(\begin{array}{cc}
V_{2}-c_{2} & p \\
p & V_{1}+c_{1}
\end{array}\right)
$$

The gap spectrum of $L_{1}$ has a close connection with $L^{2}$ which we now describe. For $d>0$, set $M_{d}(y)=L^{2} y-d^{2} y$; we say $M_{d}$ is non-oscillatory at $\infty(0)$ provided that there is a number $b>0$ such that on $(b, \infty)((0, b))$ no nontrivial solution $y$ of $M_{d} y=0$ vanishes twice, i.e., satisfies $y\left(b_{1}\right)=0=y\left(b_{2}\right)$ for $b<b_{1}<b_{2}$. Let $K_{0}$ be the minimal operator associated with $L^{2}$. By the spectral theorem for self-adjoint operators, the spectrum of a self-adjoint extension $K$ of $K_{0}$ has infinitely many points in $\left(-\infty, d^{2}\right)$ if, and only if, 
there is an infinite dimensional subspace $G \subset D(K)$ such that $(K y, y)<d^{2}(y, y)$ for all $y \in G$ [7, Section 3]. By standard arguments (cf. [7, Sections 10,12, 13]) this is equivalent to $M_{d}$ being oscillatory at either 0 or $\infty$. Note that $L_{1}^{2}$ is a self-adjoint extension of $K_{0}$. By the spectral mapping theorem [5, p. 604], $\sigma\left(L_{1}^{2}\right)=\left\{\lambda^{2}: \lambda \in \sigma\left(L_{1}\right)\right\}$; hence $\sigma\left(L_{1}\right) \cap(-d, d)$ is finite if, and only if, $\sigma\left(L_{1}^{2}\right) \cap\left(-\infty, d^{2}\right)$ is finite. Thus we have the criterion:

Theorem 2.1. For $d>0, \sigma\left(L_{1}\right) \cap(-d, d)$ is finite if, and only if, $M_{d}$ is nonoscillatory at both 0 and $\infty$.

A further useful object is the quadratic form $Q_{d}$ of $M_{d}$ given by

$$
\begin{aligned}
Q_{d}(y) & =\int_{0}^{\infty} y^{*} M_{d}(y) d x \\
& =\int_{0}^{\infty}\left[\left(y^{\prime}\right)^{*} y^{\prime}+\left(y^{\prime}\right)^{*} J P y-y^{*}(P J) y^{\prime}+y^{*}\left(P^{2}-d^{2} I\right) y\right] d x
\end{aligned}
$$

where the domain of $Q_{d}$ is all $y$ which have compact support in $(0, \infty)$, are absolutely continuous, and satisfy $\int_{0}^{\infty}\left(y^{\prime}\right)^{*} y^{\prime} d x<\infty$. The basic connection between the oscillatory properties of $M_{d}$ and $Q_{d}$ is (cf. $[4,7]$ ):

Theorem 2.2. $M_{d}$ is nonoscillatory at $\infty(0)$ if, and only if, there is a number $b>0$ such that $Q_{d}(y)>0$ for all nontrivial $y$ in domain $Q_{d}$ with support in $(b, \infty)((0, b))$.

To establish oscillation criteria for $M_{d}$ we use positive linear functionals. These were first introduced in oscillation theory by Etgen and Pawlowski [6]. A nontrivial linear functional $g$ defined on the real $n \times n$ matrices is said to be positive if $g(B) \geqq 0$ whenever $B$ is symmetric and positive semidefinite $(B \geqq 0)$. All such $g$ have the representation $g(B)=\sum_{i=1}^{k}\left(B u_{i}, u_{i}\right)$ where $u_{i}$ are non-zero $n$-vectors [cf. 17]. Recall a second-order scalar differential operator $l$ is said to be oscillatory at $\infty(0)$ if all solutions of $l(z)=0$ have infinitely many zeros in a neighborhood of $\infty(0)$.

Theorem 2.3. Let $d>0, g$ be a nontrivial positive linear functional and assume $P$ of (2.1) is locally absolutely continuous. Then $\sigma\left(L_{1}\right) \cap(-d, d)$ is infinite if the scalar differential equation,

$$
-g(I) z^{\prime \prime}+g\left(P^{2}-d^{2} I+\left[P^{\prime} J-J P^{\prime}\right] / 2\right) z=0
$$

is oscillatory either at 0 or at $\infty$.

Proof. Suppose to the contrary that $\sigma\left(L_{1}\right) \cap(-d, d)$ is finite. Let $(2.3)$ be oscillatory say at $\infty$. Then by Theorems 2.1 and 2.2 there is a number $b$ such that $Q_{d}(y)>0$ for all nontrivial $y$ in domain $Q_{d}$ with support in $(b, \infty)$. Since $(2.3)$ is oscillatory at $\infty$ there is a real nontrivial solution $z$ such that $z\left(b_{1}\right)=z\left(b_{2}\right)=0$ with $b<b_{1}<b_{2}$. Thus multiplying 
(2.3) by $z$ and integrating by parts yields

$$
\int_{b_{1}}^{b_{2}}\left[g(I)\left|z^{\prime}\right|^{2}+g\left(P^{2}-d^{2} I+\left[P^{\prime} J-J P^{\prime}\right] / 2\right) z^{2}\right] d x=0 .
$$

Thus by the above representation for $g$ there is a non-zero vector $u_{i}$ such that

$$
\int_{b_{1}}^{b_{2}}\left\{\left(u_{i}, u_{i}\right)\left|z^{\prime}\right|^{2}+\left(\left[P^{2}-d^{2} I+\left(P^{\prime} J-J P^{\prime}\right) / 2\right] u_{i}, u_{i}\right) z^{2}\right\} d x \leqq 0 .
$$

Set $y(x)=z(x) u_{i}$ on $\left[b_{1}, b_{2}\right]$ and zero elsewhere. Then $y$ is in domain $Q_{d}$ with support in $(b, \infty)$. Further, an integration by parts in (2.2) gives that

$$
Q_{d}(y)=\int_{b_{1}}^{b_{2}}\left\{u_{i}^{*} u_{i}\left|z^{\prime}\right|^{2}+u_{i}^{*}\left[\left(P^{\prime} J-J P^{\prime}\right) / 2+P^{2}-d^{2} I\right] u_{i} z^{2}\right\} d x
$$

which is the same as (2.4). Thus $Q_{d}(y) \leqq 0$ contrary to $Q_{d}(y)>0$; hence $\sigma\left(L_{1}\right) \cap(-d, d)$ is infinite.

For $g(B)=(B u, u), u=\left(\begin{array}{l}\alpha \\ \beta\end{array}\right) \neq\left(\begin{array}{l}0 \\ 0\end{array}\right)$, equation (2.3) reduces to

$$
-z^{\prime \prime}+\Gamma(x) z=0
$$

with

$$
\begin{aligned}
\Gamma(x)= & \left(\alpha^{2}+\beta^{2}\right)^{-1}\left\{\left(\alpha^{2}-\beta^{2}\right) p^{\prime}-\alpha \beta\left(V_{2}^{\prime}-V_{1}^{\prime}\right)\right. \\
& +\alpha^{2}\left(\left(V_{2}-c_{2}\right)^{2}+p^{2}-d^{2}\right)+2 \alpha \beta p\left(V_{1}+V_{2}+c_{1}-c_{2}\right)+\beta^{2}\left(\left(V_{1}+c_{1}\right)^{2}+p^{2}-d^{2}\right) .
\end{aligned}
$$

For $V_{1}=V_{2}=V$, we note the following cases of $(2.5)$ by taking $(\alpha, \beta)$ equal to $(1,0)$, $(0,1),(1, \pm 1)$ respectively and also of $(2.3)$ with $g(B)=$ trace $B$.

$$
\begin{gathered}
-z^{\prime \prime}+\left\{p^{\prime}+\left(V-c_{2}\right)^{2}+p^{2}-d^{2}\right\} z=0 \\
-z^{\prime \prime}+\left\{-p^{\prime}+\left(V+c_{1}\right)^{2}+p^{2}-d^{2}\right\} z=0 \\
-z^{\prime \prime}+(1 / 2)\left\{\left(V-c_{2}\right)^{2}+\left(V+c_{1}\right)^{2}+2 p^{2}-2 d^{2} \pm 2 p\left(2 V+c_{1}-c_{2}\right)\right\} z=0 \\
-z^{\prime \prime}+(1 / 2)\left\{\left(V-c_{2}\right)^{2}+\left(V+c_{1}\right)^{2}+2 p^{2}-2 d^{2}\right\} z=0 .
\end{gathered}
$$

Example 1. Let $V_{1}(x)=V_{2}(x)=a / x, a \neq 0, c_{1}=c_{2}=d=c$, and $p(x)=k / x+r / x^{2}$. Then (2.6) is

$$
-z^{\prime \prime}+\left\{p^{\prime}(x)+a^{2} / x^{2}-2 a c / x+p(x)^{2}\right\} z=0
$$

which is oscillatory at $\infty$ if $a>0$. Similarly (2.7) is oscillatory at $\infty$ if $a<0$. Thus $\sigma\left(L_{1}\right) \cap(-c, c)$ is infinite. 
For $c_{1}=c_{2}=d, p^{\prime}$ and $p^{2}$ being $0\left(x^{-2}\right)$ as $x \rightarrow \infty$, and $V$ of constant sign, it follows that (2.6) or (2.7) will be oscillatory at $\infty$ if $|V(x)| \leqq \varepsilon<2 c$ on some $\left[x_{0}, \infty\right)$ and $|V(x)| x^{2} \rightarrow \infty$ as $x \rightarrow \infty$. This gives Theorem 3 of [15].

It is possible to relax the differentiability requirements on $p, V_{1}$, and $V_{2}$. For the functional $g(B)=\left(B\left(\begin{array}{l}1 \\ 0\end{array}\right),\left(\begin{array}{l}1 \\ 0\end{array}\right)\right)\left(g(B)=\left(B\left(\begin{array}{l}0 \\ 1\end{array}\right),\left(\begin{array}{l}0 \\ 1\end{array}\right)\right)\right.$, equation (2.6) $((2.7))$ is obtained without assuming $V_{1}$ and $V_{2}$ differentiable. This is because the middle terms of (2.2) involving $V_{1}$ and $V_{2}$ are replaced by zero. For these same two choices of $g$, the differentiability requirement on $p$ can also be dropped by avoiding an integration by parts. In this case we get in place of (2.6) and (2.7) that if either of

$$
\begin{aligned}
& -z^{\prime \prime}-2 p z^{\prime}+\left\{\left(V_{2}-c_{2}\right)^{2}+p^{2}-d^{2}\right\} z=0, \\
& -z^{\prime \prime}+2 p z^{\prime}+\left\{\left(V_{1}+c_{1}\right)^{2}+p^{2}-d^{2}\right\} z=0,
\end{aligned}
$$

is oscillatory at $\infty$ or 0 , then $\sigma\left(L_{1}\right) \cap(-d, d)$ is infinite. For smooth $p$, these equations are less effective than (2.6) and (2.7) as is shown by the oscillation preserving substitution $w=z \exp \left\{\int \pm p\right\}$ for (2.10), (2.11) respectively.

\section{Finite gap spectra}

The middle two terms of (2.2) may be written

$$
\begin{aligned}
M I D:= & \int_{0}^{\infty}\left[\left(y^{\prime}\right)^{*} J P y-y^{*} P J y^{\prime}\right] d x \\
= & \int_{0}^{\infty}\left\{p\left[-\bar{y}_{1}^{\prime} y_{1}+\bar{y}_{2}^{\prime} y_{2}-y_{1}^{\prime} \bar{y}_{1}+y_{2}^{\prime} \bar{y}_{2}\right]-\left(V_{1}+c_{1}\right)\left(\bar{y}_{1}^{\prime} y_{2}+y_{1}^{\prime} \bar{y}_{2}\right)\right. \\
& \left.+\left(V_{2}-c_{2}\right)\left(\bar{y}_{2}^{\prime} y_{1}+y_{2}^{\prime} \bar{y}_{1}\right)\right\} d x .
\end{aligned}
$$

We need to distinguish two cases: $c_{1}=\min \left(c_{1}, c_{2}\right)$ and $c_{2}=\min \left(c_{1}, c_{2}\right)$. First suppose $c_{1}=\min \left(c_{1}, c_{2}\right)$. Let $c=\left(c_{1}+c_{2}\right) / 2$ and $\varepsilon=c-c_{1}$ so that $c_{2}=c+\varepsilon$. Using these values in (3.1) together with

$$
\int_{0}^{\infty}\left\{-c\left[\bar{y}_{1}^{\prime} y_{2}+y_{1}^{\prime} \bar{y}_{2}\right]-c\left[\bar{y}_{2}^{\prime} y_{1}+y_{2}^{\prime} \bar{y}_{1}\right]\right\} d x=\int_{0}^{\infty}-c\left[\bar{y}_{1} y_{2}+y_{1} \bar{y}_{2}\right]^{\prime} d x=0
$$

yields after integration by parts that

$$
M I D=\int_{0}^{\infty}\left\{p^{\prime}\left[\left|y_{1}\right|^{2}-\left|y_{2}\right|^{2}\right]-V_{1}\left[\bar{y}_{1}^{\prime} y_{2}+y_{1}^{\prime} \bar{y}_{2}\right]+\left(V_{2}-2 \varepsilon\right)\left[\bar{y}_{2}^{\prime} y_{1}+y_{2}^{\prime} \bar{y}_{1}\right]\right\} d x .
$$

Thus if $0<\beta_{i} \leqq 1$ for $i=1,2$, we have that

$$
Q_{d}(y)=\int_{0}^{\infty}\left\{\left(y^{\prime}\right)^{*} \Delta_{1} y^{\prime}+\left|\beta_{1} y_{1}^{\prime}-\beta_{1}^{-1} V_{1} y_{2}\right|^{2}+\left|\beta_{2} y_{2}^{\prime}-\beta_{2}^{-1}\left(V_{2}-2 \varepsilon\right) y_{1}\right|^{2}+y^{*} \Delta_{2} y\right\} d x
$$


where

$$
\begin{gathered}
\Delta_{1}=\left(\begin{array}{cc}
1-\beta_{1}^{2} & 0 \\
0 & 1-\beta_{2}^{2}
\end{array}\right), \\
\Delta_{2}=P^{2}-d^{2} I+\left(\begin{array}{cc}
p^{\prime}-\beta_{2}^{-2}\left(V_{2}-2 \varepsilon\right)^{2} & 0 \\
0 & -p^{\prime}-\beta_{1}^{-2} V_{1}^{2}
\end{array}\right) .
\end{gathered}
$$

Thus we have

$$
Q_{d}(y) \geqq \int_{0}^{\infty}\left[\left(y^{\prime}\right)^{*} \Delta_{1} y_{1}^{\prime}+y^{*} \Delta_{2} y\right] d x,
$$

and we have proved the following by Theorem 2.2 .

Theorem 3.1. Let $d>0,0<\beta_{i} \leqq 1$ for $i=1,2$. Then $\sigma\left(L_{1}\right) \cap(-d, d)$ is finite if one of the following holds.

(i) The matrix $\Delta_{2}$ for $\beta_{1}=\beta_{2}=1$ is positive definite in a neighbourhood of 0 and in a neighbourhood of $\infty$.

(ii) $0<\beta_{i}<1$ for $i=1,2$ and the matrix equation

$$
-\Delta_{1} y^{\prime \prime}+\Delta_{2} y=0
$$

is nonoscillatory at both 0 and $\infty$.

In a similar manner, we may prove the following when $c_{2}=\min \left(c_{1}, c_{2}\right)$. Set $c=$ $\left(c_{1}+c_{2}\right) / 2, \varepsilon=c-c_{2}$ (so that $\left.c_{1}=c+\varepsilon\right)$ and

$$
\Delta_{3}=P^{2}-d^{2} I+\left(\begin{array}{cc}
p^{\prime}-\beta_{2}^{-2} V_{2}^{2} & 0 \\
0 & -p^{\prime}-\beta_{1}^{-2}\left(V_{1}+2 \varepsilon\right)^{2}
\end{array}\right)
$$

Theorem 3.2. Let $d>0,0<\beta_{i} \leqq 1$ for $i=1,2$. Then $\sigma\left(L_{1}\right) \cap(-d, d)$ is finite if one of the following holds.

(i) The matrix $\Delta_{3}$ for $\beta_{1}=\beta_{2}=1$ is positive definite in a neighbourhood of 0 and in a neighbourhood of $\infty$.

(ii) $0<\beta_{i}<1$ for $i=1,2$ and the matrix equation

$$
-\Delta_{1} y^{\prime \prime}+\Delta_{3} y=0
$$

is nonoscillatory at both 0 and $\infty$.

Because of the relation (3.4), the nonoscillation of (3.5) at either 0 or $\infty$ implies the nonoscillation of $M_{d}$ at the same endpoint. Similar remarks apply to (3.7).

For the next example recall that the vector equation $-y^{\prime \prime}+\Gamma(x) y=0$ is nonoscillatory at $\infty$ if $\int_{x}^{\infty} \Gamma(s) d s$ exists and $\left\|\int_{x}^{\infty} \Gamma(s) d s\right\|_{0} \leqq 1 / 4 x$ for all $x$ sufficiently large (cf. [16]). By 
\|\|$_{0}$ we mean the operator norm on matrices where the Euclidean norm is used for vectors. We also use that if $-y^{\prime \prime}+\Gamma(x) y=0$ is nonoscillatory and $\Gamma_{1}(x) \geqq \Gamma(x)$, then $-y^{\prime \prime}$ $+\Gamma_{1}(x) y=0$ is nonoscillatory.

Example 2. Let $p, V=V_{1}=V_{2}$ be bounded on some $(0, \varepsilon)$ (hence (3.5) is nonoscillatory at 0 ) and for $x \geqq \varepsilon$, let $V(x)=a \sin x / x^{\delta}$ with $\delta>1$ and $p$ satisfy $\pm p^{\prime}(x)+p(x)^{2} \geqq a / x^{2}$ for some $a<1 / 4$ and $\left|\int_{x}^{\infty} p V\right|=o\left(x^{-1}\right)$ as $x \rightarrow \infty$. For $c_{1}=c_{2}=c=d$ and $\beta_{1}=\beta_{2}=\beta, \Delta_{2}$ of (3.5) satisfies

$$
\begin{gathered}
\Delta_{2}=\left(\begin{array}{cc}
\left(p^{\prime}+\left(1-\beta^{-2}\right) V^{2}-2 c V+p^{2}\right. & 2 p V \\
2 p V & -p^{\prime}+\left(1-\beta^{-2}\right) V^{2}+2 c V+p^{2}
\end{array}\right) \\
\geqq \widetilde{\Delta}_{2}:=\left(\begin{array}{cc}
\left(1-\beta^{-2}\right) V^{2}-2 c V+a / x^{2} & 2 p v \\
2 p V & \left(1-\beta^{-2}\right) V^{2}+2 c V+a / x^{2}
\end{array}\right) .
\end{gathered}
$$

Now each of $\int_{x}^{\infty} V^{2}, \int_{x}^{\infty} V, \int_{x}^{\infty} p V$ is $o\left(x^{-1}\right)$ as $x \rightarrow \infty$; hence for $x$ sufficiently large, $\left\|\int_{x}^{\infty} \tilde{\Delta}_{2}(s) d s\right\|_{0} \leqq a_{1} / x$ for some $a_{1}<1 / 4$. Thus $\beta$ can be chosen so that $(3.5)$ is nonoscillatory; hence $\sigma\left(L_{1}\right) \cap(-c, c)$ is finite.

For $p(x)=k / x$, similar considerations will yield Theorem 1 of [15] except for the case $k=1 / 2$.

We now consider some discrete spectrum results that do not use the operator $L^{2}$ directly.

Theorem 3.3. Let $J$ be a subinterval of $(0, \infty), U$ be an orthogonal matrix, and $d a$ positive number such that:

(i) either $U P+P^{*} U^{*} \geqq 2 d I$ on $J$ or $U P+P^{*} U^{*} \leqq-2 d I$ on $J$.

(ii) $\operatorname{Re} \int_{0}^{\infty}\left[y^{*} U J y^{\prime}\right] d x=0$ for all $y \in C_{0}(J)$.

Then for all $y \in C_{0}(J),\|L y\| \geqq d\|y\|$.

Proof. For $y \in C_{0}(J)$, we have

$$
\begin{aligned}
\|L y\|^{2}\|y\|^{2} & =\|L y\|^{2}\|U y\|^{2} \geqq\left|\left(L y, U^{*} y\right)\right|^{2} \\
& =\left|\int_{0}^{\infty}\left[y^{*} U\left(J y^{\prime}-P y\right)\right] d x\right|^{2} \\
& \geqq\left|\operatorname{Re} \int_{0}^{\infty}\left[y^{*} U\left(J y^{\prime}-P y\right)\right] d x\right|^{2} \\
& =\left|(1 / 2) \int_{0}^{\infty} y^{*}\left(U P+P^{*} U^{*}\right) y d x\right|^{2} \\
& \geqq\left|d \int_{0}^{\infty} y^{*} y d x\right|^{2}=d^{2}\|y\|^{4} .
\end{aligned}
$$


Corollary 3.1. Suppose the conditions of Theorem 3.3 hold for $J=\left[b_{2}, \infty\right)\left(J=\left(0, b_{1}\right]\right)$. Then $L^{2}-d^{2} I$ is nonoscillatory at $\infty(0)$. If the conditions hold for $J=(0, \infty)$, then $\sigma\left(L_{1}\right) \cap(-d, d)=\emptyset$ if $L_{0}=L$ and is either $\phi$ or degenerate otherwise.

Proof. For $y \in C_{0}\left(\left[b_{2}, \infty\right)\right)$ with $y \in D\left(L^{2}\right)$,

$$
\left\|L^{2} y\right\|\|y\| \geqq\left|\left(L^{2} y, y\right)\right|=\|L y\|^{2} \geqq d^{2}\|y\|^{2}
$$

or $\left\|L^{2} y\right\| \geqq d^{2}\|y\|$. Thus the minimal operator associated with $L^{2}$ on $\left[b_{2}, \infty\right)$ is bounded below by $d^{2}$. Thus a self-adjoint operator associated with $L^{2}$ on $\left[b_{2}, \infty\right)$ has a finite spectrum on $\left(-\infty, d^{2}\right)$ since it is a finite dimensional extension of the minimal operator. Thus $L^{2}-d^{2} I$ is nonoscillatory at $\infty$. Similar remarks apply to 0 . Note that $L_{1}$ is a 0-dimensional (1-dimensional) extension of $L_{0}$ if $L_{0}=L\left(L_{0} \neq L\right)$ so that $\sigma\left(L_{1}\right) \cap(-d, d)=\emptyset$ (contains at most one element).

Two special cases of Theorem 3.3 are of interest.

(A) Suppose $U=\left(\begin{array}{rr}-1 & 0 \\ 0 & 1\end{array}\right)$. Then a calculation shows (ii) of Theorem 3.3 holds. Also

$$
U P+P^{*} U=2\left(\begin{array}{cc}
c_{2}-V_{2} & 0 \\
0 & c_{1}+V_{1}
\end{array}\right)
$$

This yields the following corollaries.

Corollary 3.2. If $V_{1}$ and $V_{2}$ in (1.1) have compact support, then $\sigma\left(L_{1}\right) \cap\left(-c_{1}, c_{2}\right)$ is finite for all $p$.

Corollary 3.3. If $\left|V_{i}(x)\right| \rightarrow \infty$ as $x \rightarrow 0$ and as $x \rightarrow \infty$ for $i=1,2$ and $V_{1}(x) V_{2}(x)<0$ in both a neighbourhood of 0 and of infinity, then $\sigma\left(L_{1}\right) \cap(-d, d)$ is finite for all $d>0$, i.e., $L_{1}$ has a purely discrete spectrum.

(B) Suppose $U=\left(\begin{array}{ll}0 & 1 \\ 1 & 0\end{array}\right)$. Again (ii) of Theorem 3.3 holds and

$$
(1 / 2)\left(U P+P^{*} U^{*}\right)=\left(\begin{array}{cc}
p & v \\
v & p
\end{array}\right), \quad v:=(1 / 2)\left(V_{1}+V_{2}+c_{1}-c_{2}\right) .
$$

The eigenvalues of (3.9) are $p \pm v$. This gives

Corollary 3.4. Suppose $d>0$ and in a neighbourhood of $0(\infty), p \pm v \geqq d$ or $p \pm v \leqq-d$. Then $L^{2}-d^{2} I$ is nonoscillatory at $0(\infty)$.

\section{The shifting method}

Consider (1.1) with $c_{1}=c_{2}=c$ say,

$$
S(y)=J y^{\prime}-\left(\begin{array}{cc}
V_{2}-c & p \\
p & V_{1}+c
\end{array}\right) y, 0<x<\infty,
$$


and let $S_{1}$ be a self-adjoint operator determined by $S$. We assume the essential spectrum of $S_{1}$ is $(-\infty,-c] \cup[c, \infty)$ and that $S_{1}$ has infinitely many eigenvalues in the gap $[-c, c]$. We wish to determine which of the points $\pm c$ is a cluster point of the eigenvalues. For $0<\varepsilon<c$, set $T_{1}=S_{1}+\varepsilon I, T_{2}=S_{1}-\varepsilon I$. Then spectrum of $T_{1}\left(T_{2}\right)$ is the spectrum of $S_{1}$ moved to the right (left) $\varepsilon$ units. Note that $T_{1}$ becomes $L_{1}$ of Section 2 with $c_{1}=c-\varepsilon, c_{2}=c+\varepsilon$ and $T_{2}$ becomes $L_{1}$ with $c_{1}=c+\varepsilon, c_{2}=c-\varepsilon$.

Consider now $T_{1}$. By Section $2,-c$ is not a cluster point of the eigenvalues of $S_{1}$ if, and only if, $L^{2}-(c-\varepsilon)^{2} I$ with $c_{1}=c-\varepsilon, c_{2}=c+\varepsilon$ is nonoscillatory at both 0 and $\infty$. Thus to show $-c$ is a cluster point of eigenvalues, it suffices to show $L^{2}-(c-\varepsilon)^{2} I$ is oscillatory at one of $0, \infty$. Similar considerations apply to $T_{2}$ and $c$.

The following theorem utilizes these ideas.

Theorem 4.1. Suppose in (4.1) that $V_{1}=V_{2}=V, p$ is locally absolutely continuous, and

(i) $p(x)=0(1 / x), p^{\prime}(x)=0\left(1 / x^{2}\right)$ as $x \rightarrow \infty$,

(ii) $V(x) \rightarrow 0$ and $x^{2} V(x) \rightarrow-\infty$ as $x \rightarrow \infty$.

(iii) In a neighbourhood of $0, p(x) \geqq|V(x)|+c$ or $p(x) \leqq-c-|V(x)|$. Then $-c$ is $a$ cluster point of eigenvalues and $c$ is not.

Proof. It follows from the above remarks that $-c$ is a cluster point of eigenvalues if (2.7), with $c_{1}=c-\varepsilon=d, c_{2}=c+\varepsilon(0<\varepsilon<c)$ is oscillatory at infinity, i.e., if

$$
-z^{\prime \prime}+\left\{p^{\prime}+V^{2}+2(c-\varepsilon) V+p^{2}\right\} z=0
$$

is oscillatory. Now $V^{2}+p^{\prime}+p^{2}=V^{2}+0\left(x^{-2}\right)$ is small compared to $|V|$ by (i), (ii). Thus (4.2) is oscillatory at infinity by comparison with an oscillatory Euler equation, e.g., $-z^{\prime \prime}-\left(1 / x^{2}\right) z=0$.

To see that $c$ is not a cluster point of eigenvalues, we need to show $L^{2}-d^{2} I$ is nonoscillatory at both 0 and infinity with $d=c_{2}=c-\varepsilon, c_{1}=c+\varepsilon$. To see that $L^{2}-d^{2} I$ is nonoscillatory at 0 we apply Corollary 3.4. Then $p \pm v=p \pm(V+\varepsilon)$. Hence by (iii) either $p \pm v \geqq d$ or $p \pm v \leqq-d$; thus $L^{2}-d^{2} I$ is nonoscillatory at 0 . To show $L^{2}-d^{2} I$ is nonoscillatory at infinity we use Corollary 3.1 with $U$ as in (3.8); hence $L^{2}-d^{2} I$ is nonoscillatory at infinity if

$$
\left(\begin{array}{cc}
c_{2}-V & 0 \\
0 & c_{1}+V
\end{array}\right)=\left(\begin{array}{cc}
c-\varepsilon-V & 0 \\
0 & c+\varepsilon+V
\end{array}\right) \geqq(c-\varepsilon) I
$$

near infinity. By (ii), $-2 \varepsilon \leqq V(x)<0$ for $x$ sufficiently large thus implying (4.3).

If conditions (i)-(iii) of Theorem 4.1 hold with $x^{2} V(x) \rightarrow-\infty$ as $x \rightarrow \infty$ replaced by $x^{2} V(x) \rightarrow \infty$ as $x \rightarrow \infty$, then $c$ is a cluster point of eigenvalues and $-c$ is not.

Theorem 4.1 applies to Example 1 if either $r \neq 0$ or $|k|>|a|$.

In our final example we show both $\pm c$ may be cluster points.

Example 3. Suppose on $[1, \infty), V_{1}(x)=V_{2}(x)=(a \sin x) / x^{\delta}, 0<\delta<1, a \neq 0$ with $p^{\prime}$, $p=0\left(x^{-2}\right)$ as $x \rightarrow \infty$. Suppose also $c>1 / 2$. Then $c$ will be a cluster point of eigenvalues 
if for some $\varepsilon, 0<\varepsilon<c,(2.6)$ is oscillatory at $\infty$ with $c_{1}=c+\varepsilon, c_{2}=d=c-\varepsilon$, i.e., if

$$
-z^{\prime \prime}+\left(p^{\prime}(x)+a^{2} x^{-2 \delta} \sin ^{2} x-2 a(c-\varepsilon) x^{-\delta} \sin x+p(x)^{2}\right) z=0
$$

is oscillatory at $\infty$. We analyse (4.4) by means of a result of Read [19] for partial differential equations which in the one-dimensional case applied to $-z^{\prime \prime}+q(x) z=0$, $b \leqq x<\infty$, is:

Theorem [19]. Let $h$ be a positive, locally absolutely continuous function on $[b, \infty)$ and let $\varepsilon_{0}$ satisfy $0<\varepsilon_{0}<1$. For $\lambda \geqq 0$ define

$$
E(\lambda)=\left\{x \geqq b: \int_{b}^{x}\left[-q h-\left(h^{\prime}\right)^{2} / 4 \varepsilon_{0} h\right] d t \geqq \lambda\right\}
$$

If there exists an $\alpha>0$ and a sequence $\lambda_{k} \rightarrow \infty$ such that

$$
\left(1-\varepsilon_{0}\right)\left(\lambda_{k}+\alpha\right) \int_{E\left(\lambda_{k}\right)} h^{-1} \geqq 1
$$

for each $k$, then $-z^{\prime \prime}+q(x) z=0$ is oscillatory at $\infty$.

To apply this theorem to (4.4), we take $h(x)=x^{\delta}+k \sin x$ where $k=4(c-\varepsilon) a \varepsilon_{0}^{2}, \varepsilon_{0}=$ $1-\varepsilon$, and $\varepsilon<1$ is chosen so that $c>\varepsilon+1 / 2(1-\varepsilon)$. Take $b \geqq 1$ so that $h(x) \geqq x^{\delta} \varepsilon_{0}$ for $x \geqq b$. Then the terms of the integral in (4.5) which are not $0(1)$ as $x \rightarrow \infty$ are:

$$
\begin{gathered}
\int_{b}^{x}-a^{2} t^{-\delta} \sin ^{2} t d t=-a^{2} x^{1-\delta} / 2(1-\delta)+0(1) \\
\int_{b}^{x} 2 a(c-\varepsilon) k t^{-\delta} \sin ^{2} t d t=a(c-\varepsilon) k x^{1-\delta} /(1-\delta)+0(1) \\
\int_{b}^{x}-h^{\prime}(t)^{2} / 4 \varepsilon_{0} h(t) d t \geqq-\int_{b}^{x}\left(4 \varepsilon_{0}^{2} t^{\delta}\right)^{-1} h^{\prime}(t)^{2} d t=-k^{2} x^{1-\delta} / 8 \varepsilon_{0}^{2}(1-\delta)+0(1)
\end{gathered}
$$

Since by choice of $\varepsilon$,

$$
a(c-\varepsilon) k-a^{2} / 2-k^{2} / 8 \varepsilon_{0}^{2}=a^{2}\left[2(c-\varepsilon)^{2} \varepsilon_{0}^{2}-1 / 2\right]>0,
$$

we see that for each $\lambda, E(\lambda)$ contains an interval $\left[a_{\lambda}, \infty\right)$. Thus (4.6) holds for any $\alpha>0$ and sequence $\lambda_{k} \rightarrow \infty$. Therefore $c$ is a cluster point. Similar arguments apply to give $-c$ a cluster point. It is unclear if $\pm c$ remain cluster points of eigenvalues if $c<1 / 2$.

In the above examples we have that infinitely many eigenvalues are in the gap as a result of a sufficiently large long range potential $V(x)$. For the physically important case $p(x)=k / x$, an examination of $(2.5)$ shows the difficulty of constructing a potential whose singular behavior at zero produces infintely many eigenvalues in the gap. 
Finally, we make a comment on the more general operator

$$
T y:=\left(\begin{array}{cc}
\alpha_{1}^{-1} & 0 \\
0 & \alpha_{2}^{-1}
\end{array}\right) L y
$$

where $L$ is as in (1.1) and $\alpha_{1}, \alpha_{2}$ are positive functions. $T$ acts in the Hilbert space of functions $f$ satisfying $\int_{0}^{\infty}\left[\alpha_{1}\left|f_{1}\right|^{2}+\alpha_{2}\left|f_{2}\right|^{2}\right]<\infty$. If we make the transformation

$$
\left(\begin{array}{l}
y_{1} \\
y_{2}
\end{array}\right)(x)=\left(\begin{array}{cc}
\eta(x) & 0 \\
0 & \eta(x)^{-1}
\end{array}\right)\left(\begin{array}{l}
z_{1} \\
z_{2}
\end{array}\right)(t)
$$

where $\eta(x)=\left[\alpha_{2}(x) / \alpha_{1}(x)\right]^{1 / 4}$ and $t=\int \sqrt{\alpha_{1} \alpha_{2}} d x$, then a calculation shows $(\cdot=d / d t) T y=\lambda y$ reduces to

$$
J\left\{\dot{z}-\frac{1}{\sqrt{\alpha_{1} \alpha_{2}}}\left(\begin{array}{cc}
p+\eta^{\prime} / \eta & \left(V_{1}+c\right) \eta \\
\left(c_{2}-V_{2}\right) \eta^{-1} & -p-\eta^{\prime} / \eta
\end{array}\right) z\right\}=\lambda z .
$$

Since $\int\left(\alpha_{1}\left|y_{1}\right|^{2}+\alpha_{2}\left|y_{2}\right|^{2}\right) d x=\int\left(\left|z_{1}\right|^{2}+\left|z_{2}\right|^{2}\right) d t$, an eigenvalue problem $T y=\lambda y$ can be reduced to the type studied here.

\section{REFERENCES}

1. H. Behncke, The Dirac equations with an anomalous magnetic moment, $I I$, Lecture Notes in Mathematics 964, pp. 77-85 (Springer, Berlin-Heidelberg-New York, 1982).

2. $\mathrm{H}$. Behrens and $\mathrm{W}$. Buhring, Electron radial wave functions and nuclear beta-decay (Clarendon Press, Oxford, 1982).

3. M. S. Birman, The spectra of singular boundary problems, Matem. Sbornik 55 (1961), 125174; Amer. Math. Soc. Translations 53 (1966), 23-80.

4. W. A. Coppel, Stability and asymptotic behavior of differential equations (Heath, Boston, 1965).

5. N. Dunford and J. Schwarz, Linear Operators, Part I (Interscience, New York, 1958).

6. G. J. Etgen and J. F. Pawlowski, Oscillation criteria for second order self-adjoint differential systems, Pacific J. Math. 66 (1976), 99-110.

7. I. M. Glazman, Direct methods of qualitative spectral analysis of singular differential operators (Israel program for scientific translation, Jerusalem, 1965).

8. D. Hinton and J. K. Shaw, Absolutely continuous spectra of Dirac systems with long range, short range, and oscillating potentials, Quart. J. Math. (2), 36 (1985), 183-213.

9. D. Hinton and J. K. Shaw, Spectral properties of a separated Dirac operator (preprint).

10. D. Hinton and J. K. Shaw, Dirac systems with discrete spectra, Canad. J. Math., to appear.

11. H. Kalf, A limit-point criterion for separated Dirac operators and a little known result on Riccati's equation, Math. Z. 129 (1972), 75-82.

12. H. Kalf, U.-W. Schmincke, J. Walter and R. Wust, On the spectral theory of Schrodinger and Dirac operators with strongly singular potentials, Lecture Notes in Mathematics 448, pp. 182-226 (Springer, Berlin-Heidelberg-New York, 1975). 
13. M. Klaus, On the point spectrum of Dirac operators, Helv. Phy. Acta. 53 (1980), 453-462.

14. M. Klaus and R. Wust, Spectral properties of Dirac operators with singular potentials, $J$. Math. Anal. Appl. 72 (1979), 206-214.

15. O. I. Kurbenin, The discrete spectra of the Dirac and Pauli operators, Topics in Mathematical Physics 3, pp. 43-52 (Consultants Bureau, New York-London, 1969).

16. R. T. Lewis, Conjugate points of vector-matrix differential equations, Trans. Amer. Math. Soc. 231 (1977), 167-178.

17. R. T. Lewis and L. C. Wright, Comparison and oscillation criteria for selfadjoint vector matrix differential equations, Pacific J. Math. 90 (1980), 125-134.

18. B. M. Levitan and I. Sargsjan, Introduction to spectral theory: Selfadjoint ordinary differential operators. Translations of Mathematical Monographs 39 (Amer. Math. Soc., Providence, 1975).

19. T. T. Read, Elliptic equations without positive solutions, J. Diff. Eqs. 58 (1985), 381-390.

20. U. W. Schmincke, Distinguished selfadjoint extensions of Dirac operators, Math. Z. 129 (1972), 335-349.

21. H. Tamura, The asymptotic distribution of discrete eigenvalues for Dirac systems, J. Fac. Sci. U. Tokyo 23 (1976), 167-197.

22. E. C. Titchmarsh, On the nature of the spectrum in problems of relativistic quantum mechanics, Quart. J. Math. (2) 12 (1961), 227-240.

23. J. Weidmann, Oscillationsmethoden fur Systeme gewohnlicher Differentialgleichungen, Math. Z. 119 (1971), 349-373.

24. R. Wust, Dirac operators with strongly singular potentials, Math. $Z$. 152 (1977), 259-271.

Department of Mathematics

UNIVERSITY OF TENNESSEE

KNOXVILLE, TN 37996

Mathematics Department

WESTERN WASHINGTON UNIVERSITY

BELLINGHAM, WA 98225
Mathematics Department

UNIVERSITY OF OTTAWA

Ottawa, Ontario K1N 6N5

Mathematics Department

VIRGINIA TECH.

BlacksBurg, VA 24061 